\title{
The equiconvergence of the eigenfunction expansion for a singular version of one- dimensional Schrodinger operator with explosive factor
}

\author{
Zaki FA El-Raheem ${ }^{1 *}$ and $\mathrm{AH}$ Nasser $^{2}$
}

\footnotetext{
*Correspondence: zaki55@Alex-sci. edu.eg

${ }^{1}$ Department of Mathematics, Faculty of Education, Alexandria University, Alexandria, Egypt Full list of author information is available at the end of the article
}

\author{
Abstract \\ This paper is devoted to prove the equiconvergence formula of the eigenfunction \\ expansion for some version of Schrodinger operator with explosive factor. The \\ analysis relies on asymptotic calculation and complex integration. The paper is of \\ great interest for the community working in the area. \\ (2000) Mathematics Subject Classification \\ 34B05; 43B24; 43L10; 47E05 \\ Keywords: Eigenfunctions, Asymptotic formula, Contour integration, \\ Equiconvergence
}

\section{Introduction}

Consider the Dirichlet problem

$$
\begin{aligned}
& -y^{\prime \prime}+q(x) y=\lambda \rho(x) y \quad 0 \leq x \leq \pi \\
& \gamma(0)=0, \quad \gamma(\pi)=0
\end{aligned}
$$

where $q(x)$ is a non-negative real function belonging to $L_{1}[0, \pi], \lambda$ is a spectral parameter, and $\rho(x)$ is of the form

$$
\rho(x)=\left\{\begin{array}{c}
1 ; \quad 0 \leq x \leq a<\pi \\
-1 ; \quad a<x \leq \pi
\end{array}\right.
$$

In [1], the author studied the asymptotic formulas of the eigenvalues, and eigenfunctions of problem (1.1)-(1.2) and proved that the eigenfunctions are orthogonal with weight function $\rho(x)$. In [2], the author also studied the eigenfunction expansion of the problem(1.1)-(1.2). The calculation of the trace formula for the eigenvalues of the problem(1.1)-(1.2) is to appear. We mention here the basic definition and results from [1] that are needed in the progress of this work. Let $\phi(x, \lambda), \psi(x, \lambda)$ be the solutions of the problem (1.1)-(1.2) with the boundary conditions $\phi(0, \lambda)=0, \phi^{\prime}(0, \lambda)=1, \psi(\pi, \lambda)=0$, $\psi^{\prime}(\pi, \lambda)=1$ and let $W(\lambda)=\phi(x, \lambda) \psi^{\prime}(x, \lambda)-\psi(x, \lambda) \phi^{\prime}(x, \lambda)$ be the Wronskian of the two linearly independent solutions $\phi(x, \lambda), \psi(x, \lambda)$. It is known that $W$ is independent

(C) 2011 El-Raheem and Nasser; licensee Springer. This is an Open Access article distributed under the terms of the Creative Commons Attribution License (http://creativecommons.org/licenses/by/2.0), which permits unrestricted use, distribution, and reproduction in any medium, provided the original work is properly cited. 
of $x$ so that for $x=a$ let $W(\lambda)=\Psi(\lambda)$, the eigenvalues of (1.1)-(1.2) coincide with the roots of the equation $\Psi(\lambda)=0$, which are simple. It is easy to see that the roots of $\Psi(\lambda)=0$ are simple. The function

$$
R(x, \xi, \lambda)=\frac{1}{\Psi(\lambda)} \begin{cases}\varphi(x, \lambda) \psi(\xi, \lambda), & x \leq \xi \\ \varphi(x, \lambda) \psi(x, \lambda), & \xi \leq x\end{cases}
$$

is called the Green's function of the Dirichlet problem (1.1)-(1.2). This function satisfies for $\lambda=\lambda_{k}$ the relation

$$
R(x, \xi, \lambda)=\frac{1}{\lambda-\lambda_{k}} \frac{\varphi\left(x, \lambda_{k}\right) \psi\left(x, \lambda_{k}\right)}{a_{k}}+R_{1}(x, \xi, \lambda)
$$

where $\lambda_{k}$ are the eigenvalues of the Dirichlet problem (1.1)-(1.2) and $a_{k} \neq 0$, where $a_{k}=\int_{0}^{\pi} \rho(x) \varphi^{2}(x, \lambda) \mathrm{d} x$ are the normalization numbers of the eigenfunctions of the same problem (1.1)-(1.2). We consider now the Dirichlet problem (1.1)-(1.2) in the simple form of $q(x) \equiv 0$. For $q(x)=0$, the Dirichlet problem (1.1)-(1.2) takes the form

$$
\begin{aligned}
& -y^{\prime \prime}=\lambda \rho(x) y \quad 0 \leq x \leq \pi \\
& \gamma(0)=0, \quad y(\pi)=0 .
\end{aligned}
$$

Let the eigenfunctions of the problem (1.6) be characterized by the index "o," i.e., $\phi_{o}$ $(x, \lambda)$ and $\psi_{o}(x, \lambda)$ are the solutions of the problem (1.6) in cases of $\rho(x)=1$ and $\rho(x)$ $=-1$, respectively, where

$$
\begin{aligned}
& \varphi_{0}(x, \lambda)=\frac{\sin s x}{s} \quad 0 \leq x \leq \pi \\
& \psi_{0}(x, \lambda)=\frac{\sinh s(\pi-x)}{s} \quad a \leq x \leq \pi
\end{aligned}
$$

From (1.7), we notice that $\phi_{o}(x, \lambda) \psi_{o}(x, \lambda)$ are defined on parts of the interval $[0, \pi]$, and these formulas must be extended to all intervals $[0, \pi]$ to enable us to study the Green's function $R(x, \xi, \lambda)$ in case of $q(x) \equiv 0$. The following lemma study this extension

Lemma 1.1 The solutions $\phi_{o}(x, \lambda)$ and $\psi_{o}(x, \lambda)$ have the following asymptotic formulas

$$
\begin{aligned}
& \varphi_{o}(x, \lambda)= \begin{cases}\frac{\sin s x}{s} ; & 0 \leq x \leq a \\
-\frac{\sin s a}{s} \cosh s(x-a)-\frac{\cos s a}{s} \sinh s(x-a) ; \quad a<x \leq \pi\end{cases} \\
& \varphi_{0}(x, \lambda)= \begin{cases}-\frac{\sinh s(\pi-a)}{s} \cos s(x-a-) \frac{\cosh s(\pi-a)}{s} \sin s(x-a) ; \quad 0 \leq x \leq a \\
\frac{\sinh s(\pi-x)}{s} ; & a<x \leq \pi .\end{cases}
\end{aligned}
$$

Proof: The fundamental system of solutions of the equation $-y^{\prime \prime}=s^{2} y,(0 \leq x \leq a)$ is $y_{1}(x, s)=\sin s x, y_{2}(x, s)=\cos s x$. Similarly, the fundamental system of the equation $y^{\prime \prime}$ $=s^{2} y,(a<x \leq \pi)$ is $z_{1}(x, s)=\sinh s(\pi-x), z_{2}(x, s)=\cosh s(\pi-x)$. So that the solutions $\phi_{o}(x, \lambda)$ and $\psi_{o}(x, \lambda)$, over $[0, \pi]$, can be written in the forms

$$
\varphi_{o}(x, \lambda)=\left\{\begin{array}{l}
\frac{\sin s a}{s} ; \quad 0 \leq x \leq a \\
c_{1} z_{1}(x, s)+c_{z} z_{2}(x, s) ; \quad a<x \leq \pi .
\end{array}\right.
$$




$$
\varphi_{o}(x, \lambda)=\left\{\begin{array}{lc}
c_{3} y_{1}(x, s)+c_{4} z_{2}(x, s) ; \quad 0 \leq x \leq a \\
\frac{\sinh s(\pi-x)}{s} ; & a<x \leq \pi
\end{array}\right.
$$

The constants $c_{i}, i=1,2,3,4$ are calculated from the continuity of $\phi_{o}(x, \lambda)$ and $\psi_{o}(x$, $\lambda$ ) together with their first derivatives at the point $x=a$, from which it can be easily seen that

$$
\begin{aligned}
& c_{1}=-\frac{\sin s a}{s} \sinh s(\pi-a)-\frac{\cos s a}{s} \cosh s(\pi-a) \\
& c_{2}=\frac{\sin s a}{s} \cosh s(\pi-a)+\frac{\cos s a}{s} \sinh s(\pi-a),
\end{aligned}
$$

Substituting (1.12) into (1.10), we get (1.8). In a similar way, we calculate the constants $c_{3}, c_{4}$ where

$$
\begin{aligned}
& c_{3}=\frac{\sinh s(\pi-a)}{s} \sin s a-\frac{\cosh s(\pi-a)}{s} \cos s a \\
& c_{4}=\frac{\sinh s(\pi-a)}{s} \cos s a-\frac{\cosh s(\pi-a)}{s} \sin s a .
\end{aligned}
$$

Substituting (1.12) and (1.13) into (1.10) and (1.11), respectively, we get the required relations (1.8) and (1.9)

\section{The function $R(x, \xi, \lambda)$ and the equiconvergence}

The Green's function plays an important role in studying the equiconvergence theorem, so that, in addition to $R(x, \xi, \lambda)$, we must study the corresponding Green's function for $q(x) \equiv 0$. Let $R_{o}(x, \xi, \lambda)$ be the Green's function of problem (1.6), which is defined by

$$
R_{o}(x, \xi, \lambda)=\frac{-1}{\Psi_{o}(\lambda)}\left\{\begin{array}{l}
\varphi_{o}(x, \lambda) \psi_{o}(\xi, \lambda) x \leq \xi \\
\varphi_{o}(\xi, \lambda) \psi_{o}(x, \lambda) \xi \leq x
\end{array}\right.
$$

where the function

$$
\Psi_{o}(\lambda)=\frac{-\sin s a}{s} \cosh s(\pi-a)-\frac{\cos s a}{s} \sinh s(\pi-a)
$$

satisfies the following inequality on $\Gamma_{n}$, which is defined by $(2.21)$

$$
\left|\Psi_{o}(\lambda)\right| \geq C \frac{e^{|\operatorname{Im} s| a+|\operatorname{Re} s|(\pi-a)}}{|s|} .
$$

Following [2], we state some basic asymptotic relations that are useful in the discussion. The solutions $\phi(x, \lambda)$ and $\psi(x, \lambda)$ of the Dirichlet problem (1.1)-(1.2) have the following asymptotic formula

$$
\begin{aligned}
& \varphi(x, \lambda)=\left\{\begin{array}{l}
\frac{\sin s x}{s}+O\left(\frac{e^{|\operatorname{Im} s| x}}{\left|s^{2}\right|}\right) ; \quad 0 \leq x \leq a \\
\frac{\beta(x)}{s \beta(a)}[\sin s a \cosh s(a-x)-\cos s a \quad \sinh s(a-x)] \\
+O\left(\frac{e^{|\operatorname{Im} s| a+|\operatorname{Re} s|(a-x)}}{\left|s^{2}\right|}\right), \quad a<x \leq \pi .
\end{array}\right. \\
& \psi(x, \lambda)= \begin{cases}\frac{\alpha(x)}{s \alpha(a)}[\cos s(x-a) \sinh s(\pi-a)-\sin s(x-a) \quad \cosh s(\pi-a)] \\
+O\left(\frac{e^{\mid I m} s|(x-a)+| \operatorname{Re} s \mid(x-a)}{\left|s^{2}\right|}\right), & 0 \leq x \leq a \\
\frac{\sinh s(\pi-x)}{s}+O\left(\frac{e^{|\operatorname{Re} s|(\pi-a)}}{\left|s^{2}\right|}\right) ; & a \leq x \leq \pi .\end{cases}
\end{aligned}
$$


where

$$
\alpha(x)=\frac{1}{2} \int_{0}^{x} q(t) \mathrm{d} t, \quad \beta(x)=\frac{1}{2} \int_{0}^{x} q(t) \mathrm{d} t, \quad \lambda=s^{2} .
$$

As we introduce in (1.4), the function $R(x, \xi, \lambda)$ is the Green's function of the problem (1.1)-(1.2), and $R_{o}(x, \xi, \lambda)$ is the corresponding Green's function of the problem (1.6). In the following lemma, we prove an important asymptotic relation for the Green's function

Lemma 2.2 For $q(x) \in L_{1}(0, \pi)$ and by the help of the asymptotic formulas (2.4), (2.5) for $\phi(x, \lambda)$ and $\psi(x, \lambda)$, respectively, the Green's function $R(x, \xi, \lambda)$ satisfies the relation

$$
R(x, \xi, \lambda)=R_{o}(x, \xi, \lambda)+r(x, \xi, \lambda)
$$

where $r(x, \xi, \lambda), \lambda \in \Gamma_{n}, n \rightarrow \infty$, satisfies

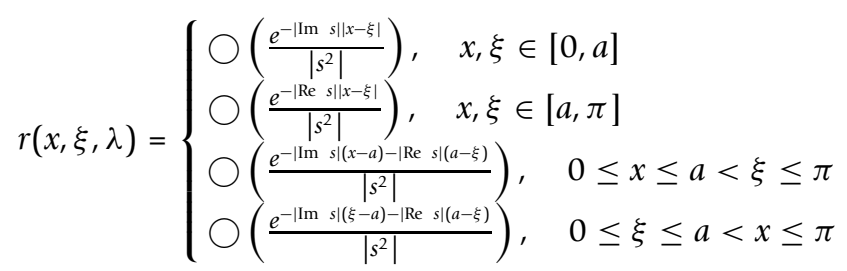

Proof: From (2.4) and (2.5), the function

$$
\Psi(\lambda)=\varphi(a, \lambda) \psi^{\prime}(a, \lambda)-\varphi^{\prime}(a, \lambda) \psi(a, \lambda)
$$

takes the form

$$
\Psi(\lambda)=\Psi_{o}(\lambda)+\bigcirc\left(\frac{e^{|l| \mathrm{m} s|a+| R e} s \mid(\pi-a)}{\left|s^{2}\right|}\right),
$$

or

$$
\Psi(\lambda)=\Psi_{o}(\lambda)\left[1+\bigcirc\left(\frac{1}{|s|}\right)\right]
$$

The function $\Psi_{o}(\lambda)$ is given by (2.2). for $x \leq \xi$, we discuss three possible cases:

(i) $0 \leq x \leq \xi \leq a$ (ii) $a \leq x \leq \xi \leq \pi$ (iii) $0 \leq x \leq a \leq \xi \leq \pi$.

The case (i) $0 \leq x \leq \xi \leq a$

From (1.4) and using (2.4) and (2.5), we have

$$
\begin{aligned}
R(x, \xi, \lambda) & =\frac{1}{\Psi(\lambda)} \varphi(x, \lambda) \psi(\xi, \lambda) \\
& =\frac{1}{\Psi(\lambda)}\left[\varphi_{0}(x, \lambda) \psi_{0}(\xi, \lambda)+\bigcirc\left(\frac{e^{\| \mid \operatorname{m~} s(a-\xi)+\text { Re } s(\pi-a)}}{|s|^{3}}\right)\right] .
\end{aligned}
$$

Using (2.9), (2.10), and (2.3), we have

$$
R(x, \xi, \lambda)=\frac{1}{\Psi_{o}(\lambda)}\left[\varphi_{o}(x, \lambda) \psi_{o}(\xi, \lambda)+\bigcirc\left(\frac{e^{|l \mathrm{~m} s|(x-\xi)}}{|s|^{2}}\right)\right] .
$$

So that from (2.1), for $0 \leq x \leq \xi \leq a$, we have

$$
R(x, \xi, \lambda)=R_{o}(x, \xi, \lambda)+\bigcirc\left(\frac{e^{|| l m} s \mid(x-\xi)}{|s|^{2}}\right)
$$

The case (ii) $a \leq x \leq \xi \leq \pi$. 
Again, from (1.4) and using (2.4) and (2.5), we have

$$
\begin{aligned}
R(x, \xi, \lambda) & =\frac{1}{\Psi(\lambda)} \varphi(x, \lambda) \psi(\xi, \lambda) \\
& =\frac{1}{\Psi(\lambda)}\left[\varphi_{0}(x, \lambda) \psi_{o}(\xi, \lambda)+\bigcirc\left(\frac{e^{|\operatorname{lm} s| a+|\operatorname{Re} s|(\pi-a+x-\xi)}}{|s|^{3}}\right)\right]
\end{aligned}
$$

Using (2.9), (2.10), and (2.3), we have

$$
R(x, \xi, \lambda)=\frac{1}{\Psi_{o}(\lambda)}\left[\varphi_{o}(x, \lambda) \psi_{o}(\xi, \lambda)+\bigcirc\left(\frac{e^{\mid \operatorname{Re} s(x-\xi)}}{|s|^{2}}\right)\right]
$$

So that from (2.1), for $a \leq x \leq \xi \leq \pi$, we have

$$
R(x, \xi, \lambda)=R_{o}(x, \xi, \lambda)+\bigcirc\left(\frac{e^{|\mathrm{Re} s|(x-\xi)}}{|s|^{2}}\right)
$$

The case (iii) $0 \leq x \leq a \leq \xi \leq \pi$.

From (1.4) and using (2.4) and (2.5), we have

$$
\begin{aligned}
R(x, \xi, \lambda) & =\frac{1}{\Psi(\lambda)} \varphi(x, \lambda) \Psi(\xi, \lambda) \\
& =\frac{1}{\Psi(\lambda)}\left[\varphi_{0}(x, \lambda) \psi_{o}(x, \lambda) \psi_{0}(\xi, \lambda)+\bigcirc\left(\frac{e^{\operatorname{IIm} s|x+| \mid R e} s(\pi-\xi)}{|s|^{3}}\right)\right]
\end{aligned}
$$

Using (2.9), (2.10), and (2.3), we have

$$
R(x, \xi, \lambda)=\frac{1}{\psi_{0}(\lambda)}\left[\varphi_{0}(x, \lambda) \psi_{o}(\xi, \lambda)+\bigcirc\left(\frac{e^{|\operatorname{Im} s|(x-a)+|\operatorname{Re} s|(a-\xi)}}{|s|^{2}}\right)\right] .
$$

So that from (2.1), for $a \leq x \leq \xi \leq \pi$, we have

$$
R(x, \xi, \lambda)=R_{o}(x, \xi, \lambda)+\bigcirc\left(\frac{e^{|\operatorname{Im} s|(x-a)+|\operatorname{Re} s|(a-\xi)}}{|s|^{2}}\right)
$$

The asymptotic formulas of $R(x, \xi, \lambda)$ in case of $\xi \leq x$ remains to be evaluated and this, in turn, consists of three cases

(i*) $0 \leq \xi \leq x \leq a$ (ii*) $a \leq \xi \leq x \leq \pi$ (iii*) $0 \leq \xi \leq a \leq x \leq \pi$.

The case (i*) $0 \leq \xi \leq x \leq a$ from (1.4) and using (2.4) and (2.5), we have

$$
\begin{aligned}
R(x, \xi, \lambda) & =\frac{1}{\Psi(\lambda)} \varphi(\xi, \lambda) \Psi(x, \lambda) \\
& =\frac{1}{\Psi(\lambda)}\left[\varphi_{0}(\xi, \lambda) \psi_{0}(x, \lambda)+\bigcirc\left(\frac{e^{|| \mathrm{m} s|(a-\xi-x)+| \operatorname{Re} s \mid(\pi-a)}}{|s|^{3}}\right)\right]
\end{aligned}
$$

Using (2.9), (2.10), and (2.3), we have

$$
R(x, \xi, \lambda)=\frac{1}{\psi_{0}(\lambda)}\left[\varphi_{0}(\xi, \lambda) \psi_{o}(x, \lambda)+\bigcirc\left(\frac{e^{|\operatorname{Im} s|(\xi-x)}}{|s|^{2}}\right)\right]
$$

So that from (2.1), for $a \leq \xi \leq x \leq a$, we have

$$
R(x, \xi, \lambda)=R_{o}(x, \xi, \lambda)+\bigcirc\left(\frac{e^{|\operatorname{Im} s|(\xi-x)}}{|s|^{2}}\right)
$$


The case (ii*) $a \leq \xi \leq x \leq \pi$ from (1.4) and using (2.4) and (2.5), we have

$$
\begin{aligned}
R(x, \xi, \lambda) & =\frac{1}{\psi(\lambda)} \varphi(\xi, \lambda) \psi(x, \lambda) \\
& =\frac{1}{\psi(\lambda)}\left[\varphi_{0}(\xi, \lambda) \psi_{o}(x, \lambda)+\bigcirc\left(\frac{e^{|\operatorname{Im} s| a+|\operatorname{Re} s|(\pi-x+\xi-a)}}{|s|^{3}}\right)\right]
\end{aligned}
$$

Using (2.9), (2.10), and (2.3), we have

$$
R(x, \xi, \lambda)=\frac{1}{\Psi_{o}(\lambda)}\left[\varphi_{o}(\xi, \lambda) \psi_{o}(x, \lambda)+\bigcirc\left(\frac{e^{|\operatorname{Re} s|(\xi-x)}}{|s|^{2}}\right)\right]
$$

So that from (2.1), for $a \leq \xi \leq x \leq \pi$, we have

$$
R(x, \xi, \lambda)=R_{o}(x, \xi, \lambda)+\bigcirc\left(\frac{e^{|\operatorname{Re} s|(\xi-x)}}{|s|^{2}}\right)
$$

The case (iii*) $0 \leq \xi \leq x \leq a \leq x \leq \pi$ from (1.4) and using (2.4) and (2.5), we have

$$
\begin{aligned}
R(x, \xi, \lambda) & =\frac{1}{\psi(\lambda)} \varphi(\xi, \lambda) \psi(x, \lambda) \\
& =\frac{1}{\psi(\lambda)}\left[\varphi_{o}(\xi, \lambda) \psi_{o}(x, \lambda)+\bigcirc\left(\frac{e^{|\operatorname{Im} s| \xi+|\operatorname{Re} s|(\pi-x)}}{|s|^{3}}\right)\right]
\end{aligned}
$$

Using (2.9), (2.10), and (2.3), we have

$$
R(x, \xi, \lambda)=\frac{1}{\psi_{o}(\lambda)}\left[\varphi_{o}(\xi, \lambda) \psi_{o}(x, \lambda)+\bigcirc\left(\frac{e^{|\operatorname{Im} s|(\xi-a)+|\operatorname{Re} s|(a-x)}}{|s|^{2}}\right)\right]
$$

So that from (2.1), for $a \leq \xi \leq x \leq a$, we have

$$
R(x, \xi, \lambda)=R_{o}(x, \xi, \lambda)+\bigcirc\left(\frac{e^{|\operatorname{Im} s|(\xi-a)+|\operatorname{Re} s|(a-x)}}{|s|^{2}}\right)
$$

Now from (2.11) and (2.14), we have

$$
R(x, \xi, \lambda)=R_{o}(x, \xi, \lambda)+\bigcirc\left(\frac{e^{-|\operatorname{Im} s|(x-\xi)}}{|s|^{2}}\right), \quad x, \xi \in[0, a]
$$

also, from (2.12) and (2.15), we have

$$
R(x, \xi, \lambda)=R_{o}(x, \xi, \lambda)+\bigcirc\left(\frac{e^{-|\operatorname{Re} s|(x-\xi)}}{|s|^{2}}\right), \quad x, \xi \in[0, \pi]
$$

As a result of the last discussion from (2.13), (2.16), (2.17), and (2.18), we deduce that $R(x, \xi, \lambda)$ obeys the asymptotic relation

$$
R(x, \xi, \lambda)=R_{o}(x, \xi, \lambda)+r(x, \xi, \lambda)
$$


where

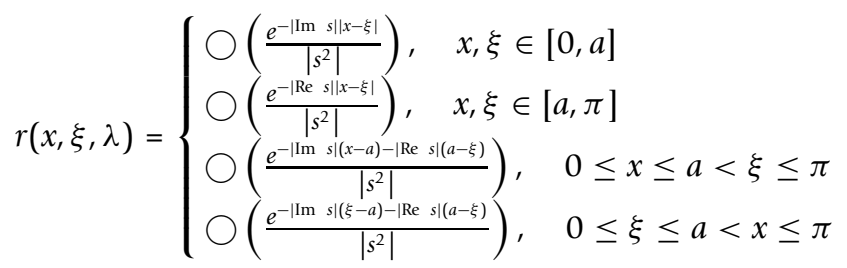

We remind here that the main purpose of this paper is to prove the equiconvergence of the eigenfunction expansion of the Dirichlet problem (1.1)-(1.2). We introduce the following notations, let $\Delta_{n, f}(x)$ denotes the $n$th partial sum

$$
\Delta_{n, f}(x)=\sum_{k=0}^{n} \frac{\varphi\left(x, \lambda_{n}^{+}\right)}{a_{k}^{+}} \int_{0}^{\pi} \rho(\xi) f(\xi) \varphi\left(\xi, \lambda_{k}^{+}\right) \mathrm{d} \xi+\sum_{k=0}^{n} \frac{\varphi\left(x, \lambda_{n}^{+}\right)}{a_{k}^{+}} \int_{0}^{\pi} \rho(\xi) f(\xi) \varphi\left(\xi, \lambda_{k}^{+}\right) \mathrm{d} \xi .
$$

where, from [1], $a_{k}^{ \pm} \neq 0$. It should be noted here, from [2], that as $n \rightarrow \infty$, the series (2.20) converges uniformly to a function $f(x) \in L_{2}(0, \pi, \rho(x))$. Let also $\Delta_{n, f}^{(o)}$ be the corresponding $n$th partial sum as (2.20), for the Dirichlet problem (1.1)-(1.2) in case of $q$ $(x) \equiv 0$. The equiconvergence of the eigenfunction expansion means that the difference $\left|\Delta_{n, f}(x)-\Delta_{n, f}^{(o)}(x)\right|$ uniformly converges to zero as $n \rightarrow \infty, x \in[0, \pi]$. In the following theorem, we prove the equiconvergence theorem of the expansions $\mid \Delta_{n, f}(x)$ and $\Delta_{n, f}^{(o)}(x) \mid$. This means that the two expansions have the same condition of convergence. Following [1], the contour $\Gamma_{n}$ is defined by

$$
\Gamma_{n}=\left\{|\operatorname{Re} s| \leq \frac{\pi}{a}\left(n-\frac{1}{4}\right)+\frac{\pi}{2 a}, \quad|\operatorname{Im} s| \leq \frac{\pi}{\pi-a}\left(n-\frac{1}{4}\right)+\frac{\pi}{2(\pi-a)}\right\} .
$$

Denote by $\Gamma_{n}^{+}$the upper half of the contour $\Gamma_{n}, \operatorname{Im} s \geq 0$, and let $L_{n}$ be the contour, in $\lambda$-domain, formed from $\Gamma_{n}^{+}$by the mapping $\lambda=s^{2}$. From (1.4), it is obvious that the poles of $R(x, \xi, \lambda)$ are the roots of the function $\Psi(s)$, which is the spectrum of the problem (1.1)-(1.2).

Theorem 2.1 Under the validity of lemma 1.1 and lemma 2.2, the following relation of equiconvergence holds true

$$
\lim _{n \rightarrow \infty} \sup _{0 \leq x \leq \pi}\left|\Delta_{n, f}(x)-\Delta_{n, f}^{(o)}(x)\right|=0 .
$$

Proof: Multiply both sides of (2.7) by $\rho(\xi) f(\xi)$ and then integrating from 0 to $\pi$, we have

$$
\int_{0}^{\pi} R(x, \xi, \lambda) \rho(\xi) f(\xi) d \xi=\int_{0}^{\pi} R_{o}(x, \xi, \lambda) \rho(\xi) f(\xi) d \xi+\int_{0}^{\pi} r(x, \xi, \lambda) \rho(\xi) f(\xi) d \xi
$$

where $f(x) \in L_{2}[0, \pi, \rho(x)]$. We multiply the last equation by $\frac{1}{2 \pi i}$ and then integrating over the contour $L_{n}$ in the $\lambda$-domain, we have 


$$
\begin{aligned}
& \frac{1}{2 \pi i} \oint_{L_{n}}\left\{\int_{0}^{\pi} R(x, \xi, \lambda) \rho(\xi) f(\xi) d \xi\right\} d \lambda \\
& =\frac{1}{2 \pi i} \oint_{L_{n}}\left\{\int_{0}^{\pi} R(x, \xi, \lambda) \rho(\xi) f(\xi) d \xi\right\} d \lambda+\frac{1}{2 \pi i} \oint_{L_{n}}\left\{\int_{0}^{\pi} r(x, \xi, \lambda) \rho(\xi) f(\xi) d \xi\right\} d \lambda .
\end{aligned}
$$

From equation (1.5), we have the following

$$
\operatorname{Res}_{\lambda=\lambda_{k}^{ \pm}} R(x, \xi, \lambda)=\frac{\varphi\left(x, \lambda_{k}^{ \pm}\right) \varphi\left(\xi, \lambda_{k}^{ \pm}\right)}{a_{k}^{ \pm}}
$$

Applying Cauchy residues formula to the first integral of (2.23) and using (2.24), we have

$$
\begin{aligned}
& \frac{1}{2 \pi i} \oint_{L_{n}}\left\{\int_{0}^{\pi} R(x, \xi, \lambda) \rho(\xi) f(\xi) \mathrm{d} \xi\right\} d \lambda=\sum_{k=0}^{n} \operatorname{Res}_{\lambda=\lambda_{k}^{ \pm}}\left\{\int_{0}^{\pi} R\left(x, \xi, \lambda_{k}^{ \pm}\right) \rho(\xi) f(\xi) \mathrm{d} \xi\right\} \\
& =\sum_{k=0}^{n} \frac{\varphi\left(x, \lambda_{n}^{+}\right)}{a_{k}^{+}} \int_{0}^{\pi} \rho(\xi) f(\xi) \varphi\left(\xi, \lambda_{k}^{+}\right) \mathrm{d} \xi+\sum_{k=0}^{n} \frac{\varphi\left(x, \lambda_{n}^{+}\right)}{a_{k}^{+}} \int_{0}^{\pi} \rho(\xi) f(\xi) \varphi\left(\xi, \lambda_{k}^{+}\right) \mathrm{d} \xi .=\Delta_{n, f}(x)
\end{aligned}
$$

Similarly, we carry out the same procedure to the second integral of (2.23) and we get an expression analogous to (2.25)

$$
\frac{1}{2 \pi i} \oint_{L_{n}}\left\{\int^{\pi} R_{o}(x, \xi, \lambda) \rho(\xi) f(\xi) \mathrm{d} \xi\right\} d \lambda=\Delta_{n, f}^{(o)}(x) .
$$

So that from (2.25), (2.26), and (2.23), we get

$$
\Delta_{n, f}(x)-\Delta_{n, f}^{(o)}(x)=\frac{1}{2 \pi i} \oint_{L_{n}}\left\{\int_{0}^{\pi} r(x, \xi, \lambda) \rho(\xi) f(\xi) \mathrm{d} \xi\right\} \mathrm{d} \lambda,
$$

from which it follows that

$$
\left|\Delta_{n, f}(x)-\Delta_{n, f}^{(o)}(x)\right| \leq \frac{1}{2 \pi} \oint_{L_{n}}\left\{\int_{0}^{\pi}|r(x, \xi, \lambda)||f(\xi)| \mathrm{d} \xi\right\} \mathrm{d}|\lambda| .
$$

The last Equation (2.27) is an essential relation in the proof of the theorem, because the theorem is established if we prove that $\frac{1}{2 \pi} \oint_{L_{n}}\left\{\int_{0}^{\pi}|r(x, \xi, \lambda)||f(\xi)| d \xi\right\} d|\lambda|$ tends to zero uniformly, $x \in[0, \pi]$. We use the same technique as in [3] We have

$$
\begin{aligned}
& \oint_{L_{n}}\left\{\int_{0}^{\pi}|r(x, \xi, \lambda)||f(\xi)| \mathrm{d} \xi\right\}|\mathrm{d} \lambda| \\
& \oint_{L_{n}}\left\{\int_{0}^{\pi}|r(x, \xi, \lambda)||f(\xi)| \mathrm{d} \xi\right\}|\mathrm{d} \lambda|+\oint_{L_{n}}\left\{\int_{0}^{\pi}|r(x, \xi, \lambda)||f(\xi)| \mathrm{d} \xi\right\}|\mathrm{d} \lambda| \\
& \leq M_{1} \oint_{L_{n}}\left\{\int_{0}^{a} \frac{e^{|\operatorname{lm} \lambda||x-\xi|}}{|s|^{2}}|f(\xi)| \mathrm{d} \xi\right\}|\mathrm{d} \lambda|+M_{2} \oint_{L_{n}}\left\{\int_{0}^{a} \frac{e^{|\operatorname{lm} \lambda|(a-x)-|\operatorname{Re} \lambda|(\xi-a)}}{|s|^{2}}|f(\xi)| \mathrm{d} \xi\right\}|\mathrm{d} \lambda|
\end{aligned}
$$


where $M_{1}$ and $M_{2}$ are constants.

We treat now the integral $\int_{0}^{a}$ in (2.30). Let $\delta>0$ be a sufficiently small number and let $\lambda=s^{2}$, so that, for $x, \xi \in[0, a]$, we have

$$
\begin{aligned}
& \oint_{L_{n}}\left\{\int_{0}^{q} \frac{e-|\operatorname{Im} \lambda||x-\xi|}{|s|^{2}}|f(\xi) d \xi|\right\}|d \lambda| \\
& =\int_{\Gamma_{n}^{+}} \frac{|\mathrm{d} s|}{|s|}\left\{\int_{|x-\xi| \leq \delta} e^{-|\operatorname{Im} \lambda||x-\xi|}|f(\xi)| d \xi+\int_{|x-\xi| \leq \delta} e^{-|\operatorname{Im} \lambda||x-\xi|}|f(\xi)| d \xi\right\} \\
& \leq \int_{\Gamma_{n}^{+}} \frac{|\mathrm{d} s|}{|s|} \int_{|x-\xi| \leq \delta}|f(\xi)| d \xi+\int_{0}^{\pi}|f(\xi)| d \xi \int_{\Gamma_{n}^{+}} e^{-|\operatorname{Im} \lambda| \delta \frac{|\mathrm{d} s|}{|s|}} \\
& \leq 4 \int_{|x-\xi| \leq \delta}|f(\xi)| d \xi+\int_{0}^{\pi}|f(\xi)| d \xi\left[\frac{2}{\delta\left(n-\frac{1}{4}\right)}+2 e^{-\delta\left(n-\frac{1}{4}\right)}\right] .
\end{aligned}
$$

This means that

$$
M_{1} \oint_{L_{n}}\left\{\int_{0}^{a} \frac{e^{-|\operatorname{Im} \lambda||x-\xi|}}{|s|^{2}}|f(\xi)| d \xi\right\}|d \lambda| \leq C_{1} \int_{|x-\xi| \leq \delta}|f(\xi)| d \xi+\frac{C_{2}}{\delta_{n}}+C_{3} e^{-\delta n}
$$

where $C_{1}, C_{2}$, and $C_{3}$ are independent of $x, n$ and $\delta$. In a similar way, we estimate the second integral $\int_{a}^{\pi}$ in (2.30) in the form

$$
M_{2} \oint_{L_{n}}\left\{\int_{0}^{\pi} \frac{e^{-|I m \lambda|(a-x)-}|\operatorname{Re} \lambda|(\xi-a)}{|s|^{2}}|f(\xi)| d \xi\right\}|d \lambda| . \leq C_{1}^{*} \int_{|x-\xi| \leq \delta}|f(\xi)| d \xi+\frac{C_{2}^{*}}{\delta n}+C_{3}^{*} e^{-\delta n}
$$

where $C_{1}^{*}, C_{2}^{*}$, and $C_{3}^{*}$ are independent of $x, n$, and $\delta$. Substituting (2.30) and (2.31) into (2.28) and using (2.29), we have

$$
\left|\Delta_{n, f}(x)-\Delta_{n, f}^{(o)}(x)\right| \leq A \int_{|x-\xi| \leq \delta}|f(\xi)| d \xi+\frac{B}{\delta n}+C e^{-\delta n}
$$

where $\mathrm{A}, \mathrm{B}$, and $\mathrm{C}$ are constants independent of $x, n$, and $\delta$. We apply now the property of absolute continuity of Lesbuge integral to the function $f(x) \in L_{1}[0, \pi]$.

$\forall \epsilon>0, \exists \delta>0$ is sufficiently small such that $\int_{|x-\xi| \leq \delta}|f(\xi)| \mathrm{d} \xi \leq \epsilon$, where $\epsilon$ is independent of $x$ (the set $\{\xi:|x-\xi| \leq \delta\}$ is measurable). Fixing $\delta$ in (2.32), there exists $N$ such that for all $n>N, \frac{1}{\delta n}<\varepsilon$ and $e^{-\delta n}<\epsilon$, so that (2.32) takes the form

$$
\left|\Delta_{n, f}(x)-\Delta_{n, f}^{(o)}(x)\right| \leq(A+B+C) \varepsilon, \quad n>N .
$$

Since $\epsilon$ is sufficiently small as we please, it follows that $\left|\Delta_{n, f}(x)-\Delta_{n, f}^{(o)}(x)\right| \rightarrow 0$ as $n \rightarrow \infty$, uniformly with respect to $x \in[0, \pi]$, which completes the proof. 


\section{The conclusion and comments}

It should be noted here that, the theorem of equiconvergence of the eigenfunction expansion is one of interesting analytical problem that arising in the field of spectral analysis of differential operators, see [4-6]. In [3], the author studied the equiconvergence theorem of the problem

$$
\begin{aligned}
& -y^{\prime \prime}+q(x) y=\mu \rho(x) y \quad 0 \leq x \leq \pi \\
& y^{\prime}(0)-h y(0)=0 \\
& y^{\prime}(\pi)+H y(\pi)=0
\end{aligned}
$$

There are many differences between problems (3.34)-(3.35) and the present one (1.1)-(1.2), and the differences are as follows:

1- The boundary conditions of (3.35) is separated boundary conditions, whereas (1.2) is the Dirichlet-Dirichlet condition

2- The eigenfunctions of (3.34)-(3.35) is given by

$$
\varphi(x, \mu)=\left\{\begin{aligned}
\cos \lambda x & +O\left(\frac{e^{|\operatorname{Im} \lambda| x}}{|\lambda|}\right), \quad 0 \leq x \leq a \\
\cos \lambda a & \cosh \lambda(a-x)+\sin \lambda a \sinh \lambda(a-x) \\
& +O\left(\frac{e^{|\operatorname{Im} \lambda| a+|\operatorname{Re} \lambda(x-a)|}}{|\lambda|}\right), \quad a<x \leq \pi,
\end{aligned}\right.
$$

and

$$
\varphi(x, \mu)=\left\{\begin{array}{c}
\cos \lambda(\pi-a) \cos \lambda(a-x)+\sinh \lambda(\pi-a) \quad \sin \lambda(a-x) \\
\quad+O\left(\frac{e^{|\operatorname{Im} \lambda|(a-x)+|\operatorname{Re} \lambda|(\pi-x)}}{|\lambda|}\right), \quad 0 \leq x \leq a \\
\cos \lambda(\pi-a)+O\left(\frac{e^{|\operatorname{Im} \lambda| x}}{|\lambda|}\right), \quad a<x \leq \pi
\end{array}\right.
$$

3- The contour of integration is of the form

$$
\Gamma_{n}=\left\{\lambda:|\operatorname{Re} \lambda| \leq \frac{\pi}{a}\left(n+\frac{1}{4}\right)+\frac{\pi}{2 a}, \quad|\operatorname{Im} \lambda| \leq \frac{\pi}{\pi-a}\left(n+\frac{1}{4}\right)+\frac{\pi}{2(\pi-a)}\right\} .
$$

4- The remainder function $r(x, \xi, \lambda)$ admits the following inequality for $\lambda \in \Gamma_{n}, n \rightarrow$ $\infty$.

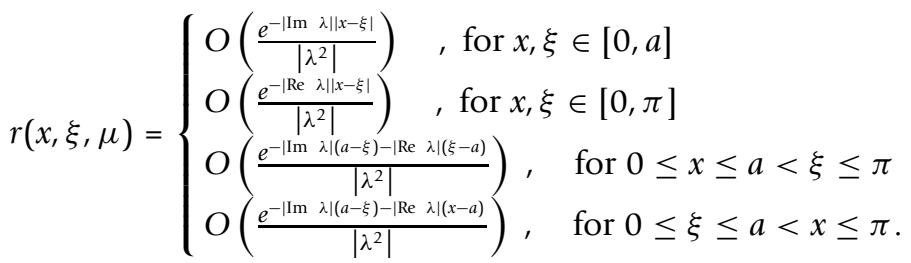

Although there are four differences between the two problems, we find that the proof of the equiconvergence formula $\left|\Delta_{n, f}(x)-\Delta_{n, f}^{(o)}(x)\right| \rightarrow 0$ as $n \rightarrow \infty$ is similar. So as long as the proof of the equiconvergence relation is carried out by means of the contour integration, we obtain the uniform convergence of the series (2.20) 


\section{Acknowledgements}

We are indebted to an anonymous referee for a detailed reading of the manuscript and useful comments and suggestions, which helped us improve this work. This work was supported by the research center of Alexandria University.

\section{Author details}

'Department of Mathematics, Faculty of Education, Alexandria University, Alexandria, Egypt ${ }^{2}$ Faculty of Industrial Education, Helwan University, Cairo, Egypt

\section{Authors' contributions}

The two authors typed read and approved the final manuscript also they contributed to each part of this work equally.

\section{Competing interests}

The authors declare that they have no competing interests.

Received: 7 June 2011 Accepted: 23 November 2011 Published: 23 November 2011

\section{References}

1. El-Raheem, ZF, Nasser, AH: On the spectral property of a Dirichlet problem with explosive factor. J Appl Math Comput. 138, 355-374 (2003). doi:10.1016/S0096-3003(02)00134-0

2. El-Raheem, ZF, Nasser, AH: The eigenfunction expansion for a Dirichlet problem with explosive factor. J Abstract Appl Anal 16 (2011). 2011, Article ID 828176

3. El-Raheem, ZF: Equiconvergence of the eigenfunctions expansion for some singular Sturm-Liouville operator. J Appl Anal. 81, 513-528 (2002). doi:10.1080/0003681021000004573

4. Darwish, A: On the equiconvergence of the eigenfunction expansion of a singular boundary value problem.Az,NEENTE, No.96AZ-D,1983.

5. Nimark, MA: The study of eigenfunction expansion of non-self adjoint differential operator of the second order on the half line. Math Truda. 3, 1891-270 (1954)

6. Saltykov, GE: On equiconvergence with Fourier integral of spectral expansion related to the non- Hermitian StormLiouville operator. pp. 18-27. ICM, Berlin (1998)

doi:10.1186/1687-2770-2011-45

Cite this article as: El-Raheem and Nasser: The equiconvergence of the eigenfunction expansion for a singular version of one-dimensional Schrodinger operator with explosive factor. Boundary Value Problems 2011 2011:45.

\section{Submit your manuscript to a SpringerOpen ${ }^{\odot}$} journal and benefit from:

Convenient online submission

Rigorous peer review

- Immediate publication on acceptance

- Open access: articles freely available online

- High visibility within the field

- Retaining the copyright to your article

Submit your next manuscript at $\boldsymbol{s p r i n g e r o p e n . c o m ~}$ 